\title{
Marchiafava-Bignami Disease: Two Chronologically Distinct Stages in the Same Patient
}

\author{
Miguel Quintas-Neves (1D, José Manuel Amorim, João Paulo Soares-Fernandes
}

Keywords: Marchiafava-Bignami disease, Magnetic resonance imaging

doi:10.1017/cjn.2020.86

Can J Neurol Sci. 2020; 47: 689-690

A 43-year-old man with chronic alcoholism came to the emergency department due to a 1-week history of severe asthenia, memory loss, and dizziness, associated with weight loss ( $8 \mathrm{~kg}$ in the previous 4 months). Neurological examination revealed temporal disorientation, scarce speech, perseveration, and bradykinesia. Brain magnetic resonance imaging (MRI) showed symmetrical lesions in the rostrum, genu, body, and splenium of the corpus callosum, which were hyperintense on fluid-attenuated inversion recovery sequence, and showed restricted diffusion (Figure 1), much more pronounced in the splenium. These findings were consistent with MarchiafavaBignami disease (MBD). Intravenous thiamine (500 mg, three times a day), oral oxazepam, and folic acid were given, with progressive clinical improvement. The patient was discharged and referred to a specialized appointment in alcohol dependence recovery.

MBD is a rare disorder that can be associated with all types of alcoholic beverages, and even with malnourished nonalcoholic patients. ${ }^{1}$ The disease typically affects the body of the corpus callosum, followed by the genu, and ultimately the splenium,, 2 being also possible the involvement of the entire corpus callosum. ${ }^{1}$

MRI plays a key role in the diagnosis of $\mathrm{MBD}$, and in distinguishing it from other diseases, as in this case lesions typically affect the central layers of the corpus callosum and are remarkably symmetric ${ }^{3}$; they are hyperintense on $\mathrm{T} 2$-weighted sequences and might show restricted diffusion due to the presence of cytotoxic edema/active fiber demyelination. ${ }^{4}$

The peculiar finding in our patient was the detection of two chronologically distinct stages: a subacute lesion extending along the rostrum, genu, and body of the corpus callosum (i.e., with less pronounced restricted diffusion), and an acute lesion in the splenium (i.e., with more pronounced restricted diffusion). Interestingly, tractography showed selective focal distortion of the posterior commissural fibers, and spectroscopy revealed a lactate peak at $1.3 \mathrm{ppm}$ (Figure 2); findings that were consistent with an active demyelination process in the splenium. ${ }^{4}$

With the assumption of successful alcoholic cessation, the preserved $\mathrm{N}$-acetylaspartate/creatine ratio - a marker of neuronal integrity - argues against necrotic lesions and might be an indicator of good prognosis to our patient. Nine months after the episode, the patient was reevaluated by the psychiatrist and had maintained alcoholic abstinence, being capable of doing all of his daily activities.
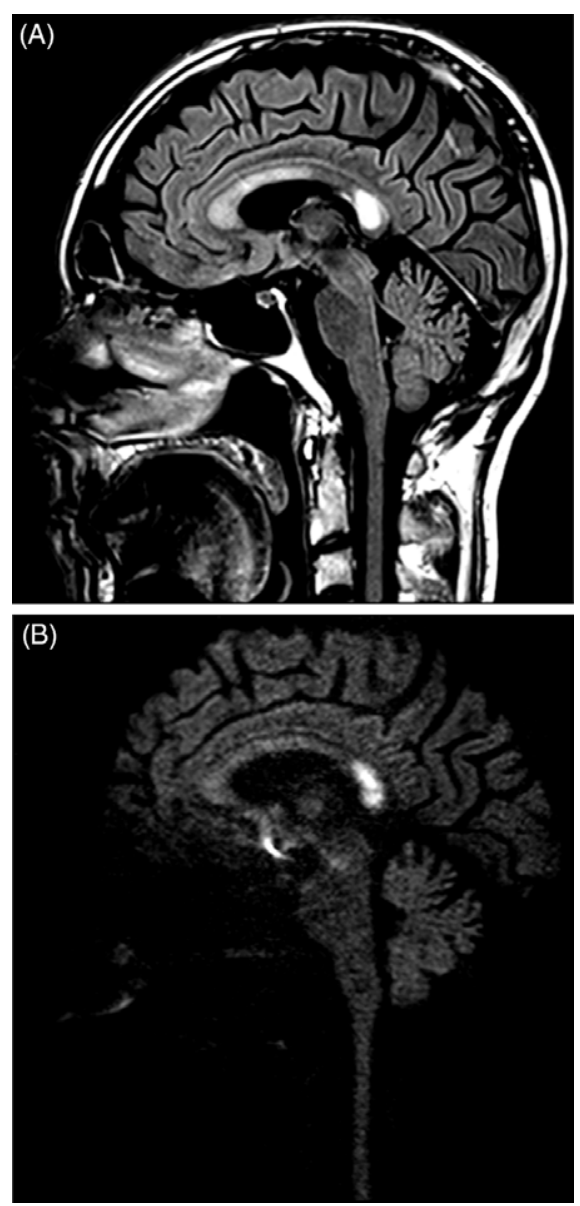

Figure 1: Brain magnetic resonance imaging shows symmetrical lesions involving the central layers of the rostrum, genu, body, and splenium of the corpus callosum; they show hyperintense signal on fluid-attenuated inversion recovery sequence (A), and restrict on diffusion-weighted imaging (B). A more pronounced restricted diffusion can be seen in the splenium.

From the Neuroradiology Department, Hospital de Braga, Braga, Portugal (MQN, JMA, JPSF)

Received September 6, 2019. Final Revisions Submitted March 25, 2020. Date of ACCEPTANCE APRIL 23, 2020.

Correspondence to: Miguel Quintas-Neves, Neuroradiology Department, Hospital de Braga, Sete Fontes, S. Vítor, 4710-243 Braga, Portugal. Email: mlqneves@gmail.com 

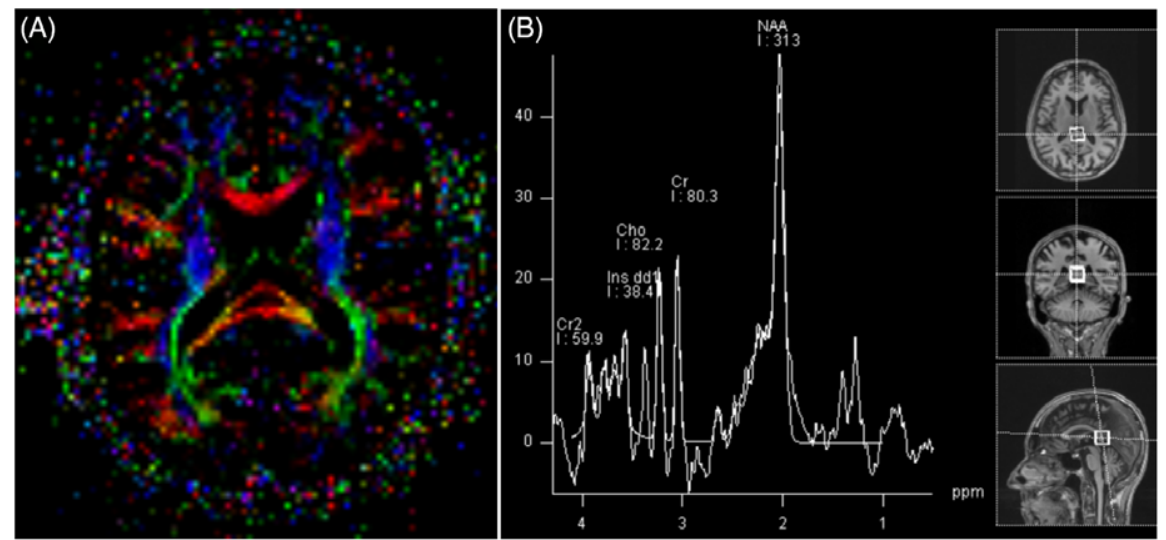

Figure 2: Tractography shows selective distortion of the posterior commissural fibers (A), and spectroscopy (Time of Echo $=30 \mathrm{~ms}$ ) reveals a lactate peak at $1.3 \mathrm{ppm}$ and a relatively preserved $\mathrm{N}$-acetylaspartate/creatine ratio $(B)$ in the splenium of the corpus callosum.

\section{Disclosures}

None.

\section{Statement of Authorship}

MQ-N: acquisition of data, clinical and imaging data review, literature review, and final manuscript writing. JMA: acquisition of data, imaging data review, and literature review. JPS-F: clinical data review, literature review, final manuscript writing, and important intellectual contribution.

\section{ETHICS INFORMATION}

This study complies with the Declaration of Helsinki and was conducted in accordance to the local ethics committee requirements. No figures or videos contain information which allows patient identification, and therefore, written patient consent for the publication was not obtained.

\section{REFERENCES}

1. Hillbom M, Saloheimo P, Fujioka S, Wszolek ZK, Juvela S, Leone MA. Diagnosis and management of Marchiafava-Bignami: a review of CT/MRI confirmed cases. J Neurol Neurosurg Psychiatry 2014;85(2):168-73.

2. Chang KH, Cha SH, Han MH. Marchiafava-Bignami disease: serial changes in corpus callosum on MRI. Neuroradiology 1992;34(6): 480-82.

3. Friese SA, Bitzer M, Freudenstein D, Voigt K, Kuker W. Classification of acquired lesions of the corpus callosum with MRI. Neuroradiology 2000;42(11):795-802.

4. Gass A, Birtsch C, Oster M, Schwartz A, Hennerici MG. Marchiafava-Bignami disease: reversibility of neuroimaging abnormality. J Comput Assist Tomogr. 1998;22(3):503-4. 